\title{
The proportion of soil-borne pathogens increases with warming at the global scale
}

Authors: Manuel Delgado-Baquerizo ${ }^{1}$, Carlos A. Guerra ${ }^{2-3}$, Concha Cano-Díaz $^{4}$, Eleonora Egidi ${ }^{5-}$ ${ }^{6}$, Jun-Tao Wang ${ }^{5-7}$, Nico Eisenhauer ${ }^{2,8}$, Brajesh K. Singh ${ }^{5-6}$, Fernando T. Maestre ${ }^{9,10}$.

\section{Affiliations:}

${ }^{1}$ Departamento de Sistemas Físicos, Químicos y Naturales, Universidad Pablo de Olavide, 41013 Sevilla, Spain.

${ }^{2}$ German Centre for Integrative Biodiversity Research (iDiv) Halle-Jena-Leipzig, Deutscher Platz 5e, 04103 Leipzig, Germany

${ }^{3}$ Institute of Biology, Martin-Luther University Halle Wittenberg, Am Kirchtor 1, 06108 Halle (Saale), Germany.

${ }^{4}$ Departamento de Biología y Geología, Física y Química Inorgánica, Escuela Superior de Ciencias Experimentales y Tecnología, Universidad Rey Juan Carlos, Calle Tulipán Sin Número, Móstoles $15 \quad$ 28933, Spain.

${ }^{5}$ Global Centre for Land-Based Innovation, Western Sydney University, Penrith South DC, NSW 2751, Australia.

${ }^{6}$ Hawkesbury Institute for the Environment, Western Sydney University, Penrith, New South Wales 2751, Australia.

${ }^{7}$ State Key Laboratory of Urban and Regional Ecology, Research Center for Eco-Environmental Sciences, Chinese Academy of Sciences, Beijing 100085, China.

${ }^{8}$ Leipzig University, Institute of Biology, Deutscher Platz 5e, 04103 Leipzig, Germany.

${ }^{9}$ Instituto Multidisciplinar para el Estudio del Medio "Ramón Margalef", Universidad de Alicante, Carretera de San Vicente del Raspeig s/n, 03690 San Vicente del Raspeig, Alicante, Spain.

${ }^{10}$ Departamento de Ecología, Universidad de Alicante, Carretera de San Vicente del Raspeig s/n, 03690 San Vicente del Raspeig, Alicante, Spain. 
Understanding the present and future distribution of soil-borne plant pathogens is critical for supporting food and fibre production in a warmer world. Using data from a global field survey and a nine-year field experiment, we show that warmer temperatures increase the relative abundance of soil-borne potential fungal plant pathogens. Moreover, we provide a global atlas of these organisms along with future distribution projections under different climate change and land use scenarios. These projections show an overall increase in the relative abundance of potential plant pathogens worldwide. This work advances understanding of the global distribution of potential fungal plant pathogens and their sensitivity to ongoing climate and land-use changes, which is fundamental to reduce their incidence and impacts on terrestrial ecosystems globally.

Around $15 \%$ of the global crop production is lost to biological threats ${ }^{1-5}$, a percentage that is expected to increase with ongoing global warming and the associated intensification of pest incidence ${ }^{1}$. This will jeopardize food security and reduce the productivity and health of terrestrial plant communities worldwide ${ }^{4}$. Many of the most aggressive plant pathogens are soil-borne fungi (e.g., Alternaria alternata or Fusarium oxysporum $)^{6-8}$ that threaten food security as the chemical 55 fungicides currently used against them are mostly ineffective ${ }^{6-8}$. In recent years, information on the distribution of plant diseases has increasingly become available at the local and regional scale (e.g., via PlantWise, https://www.plantwise.org). Moreover, the fundamental study in ref. ${ }^{9}$, provided important insights on the distribution of global fungi. Yet, global atlases of the current and future distribution of plant pathogens under contrasting global change scenarios, and based on multiple contrasting climates and vegetation types, are still lacking.

Soils from natural ecosystems provide an array of potential reservoirs for fungal pathogens surrounding croplands worldwide, challenging their productivity ${ }^{6-8}$. Moreover, natural ecosystems, which provide essential services (e.g. timber and livestock production) ${ }^{10-11}$ to billions of people, are also highly sensitive to the incidence of fungal pests ${ }^{1-6,10}$. Understanding the current 65 and future distribution of plant pathogens in natural ecosystems and the environmental factors influencing them is critical for forecasting their impact on human well-being and ecosystem sustainability under projected climate and land-use change scenarios. This could readily be seen as temperatures continue to rise along this century ${ }^{3,12}$, which might have an impact upon the proportion of potential plant pathogens worldwide. Temperature is known to determine the distribution of soil microbial communities ${ }^{9,13}$ as well as to influence the distributions of fastgrowing opportunistic fungal and animal pests ${ }^{14}$. Even so, the potential role of warming in the relative abundance of fungal plant pathogens in the soil reservoir remains largely unexplored.

Here, we used a global field survey ${ }^{15}$ conducted across 235 natural ecosystems from six continents (Supplementary Fig. 1) and a nine-year warming field experiment ${ }^{16}$ to evaluate how temperature $^{17}$ regulates the relative abundance of soil-borne potential fungal plant pathogens (potential plant pathogens hereafter). This global survey was previously used to identify the top dominant fungal phylotypes in soils across the globe ${ }^{15}$. Here, we generated global atlases for the current and future distribution of potential plant pathogens under contrasting global change scenarios, and explored causal relationships between their relative abundance and warming. Our global field survey (Methods) included a wide variety of vegetation, climates and soil types, and covered $\sim 73 \%$ of the environmental conditions found on Earth (Supplementary Appendix 1).

Using amplicon sequencing for the ITS gene, we identified 2,735 fungal phylotypes classified as potential plant pathogens out of the 23,399 fungal phylotypes found in our global survey (Supplementary Data 1$)^{6}$. Together, potential pathogenic phylotypes represented between 
0.5 and $46.5 \%$ (with the average at $14.4 \%$ ) of all ITS sequences at a given site (Fig. 1A), and included multiple potential plant pathogens with single (plant pathogens only, 37.1\% of all pathogenic phylotypes; e.g., Venturia spp.) and mixed (plant pathogen and endophyte and/or saprotrophic fungi, 62.8\% of all pathogenic phylotypes; e.g., Fusarium spp.) trophic modes (Supplementary Fig. 2; Supplementary Data 1). Our results thus indicate that soil-borne potential plant pathogens can be relatively abundant in soils from natural ecosystems worldwide. This was particularly the case in tropical and dry forests, but not in boreal and cold forests (Fig. 1B). On average, surveyed soils were dominated by a few genera of potential plant pathogens, including Alternaria, Fusarium, Venturia and Phoma (Fig. 1C; Supplementary Data 1 for a complete list), which together accounted for almost half (43.0\%) of the retrieved ITS sequences classified as potential plant pathogens. Many of these soil-borne fungal taxa include economically important potential pathogens, as they are likely to affect the health and productivity of many important crops (e.g., wheat, sunflowers, cabbages, tomatoes, and potatoes), gardening and cosmetic/medicinal plants (e.g., Hibiscus, Aloe vera), and wild species that are an important food source for livestock ${ }^{6-}$ $8,18-19$

100 We then used Structural Equation Modelling (SEM; Supplementary Figs. 3-5; Supplementary Tables 1-8) to identify the direct and indirect (e.g., via changes in soil properties and vegetation) associations between temperature and the relative abundance of potential plant pathogens across the globe. We found that mean annual temperature (MAT) had the largest positive and significant direct association with the relative abundance of soil pathogens globally (Fig. 1D; see all considered associations in Supplementary Fig. 3 and Supplementary Table 2). We also detected multiple indirect effects of MAT on the relative abundance of soil-borne potential plant pathogens via changes in vegetation types (forests and grasslands; Fig. 1D). Similar results were observed when calculating the relative abundance of potential plant pathogens from rarefied abundance (Supplementary Tables 3 and 8), when considering the relative abundance of potential 110 plant pathogens with single and mixed trophic modes (Supplementary Tables 4-5 and 8), and when focusing on probable and highly probable pathogens only (Supplementary Tables 6-8). Our analyses further indicated that MAT was the most important factor influencing the relative abundance of soil-borne potential plant pathogens globally when considering both direct and indirect effects simultaneously (total standardised effects; Fig. 1E and Supplementary Fig. 4). We

115 also found that MAT had a total positive effect on the relative abundance of fungal pathogens when focusing on the most abundant potential pathogen genera (Alternaria, Fusarium, Venturia and Phoma; Supplementary Fig. 5). Additional correlation analyses suggested that MAT is positively associated with the relative abundance of multiple genera classified as potential plant pathogens, which were found to be ubiquitous in soils across the globe ( $>50 \%$ of all locations) 120 (Fig. 2; Supplementary Data 1). Likewise, ecosystem type (e.g. forests and grasslands) and plant cover were significantly associated with the relative abundance of plant pathogens. These findings suggest that changes in land use -as those predicted with global change ${ }^{20}$ - might also alter the relative abundance of soil-borne potential pathogens globally. Other predominant environmental factors associated with specific pathogen genera include precipitation and soil pH (Fig. 2).

Together, findings from our observational survey ${ }^{15}$ suggest that increasing temperature may cause increases in the presence of potential fungal plant pathogens in soils, which might act as reservoirs of infection. Natural areas are often surrounded by croplands across the globe, and there is significant "spill over" of soil microbes between them ${ }^{21}$. Given the high dispersal abilities of fungi ${ }^{22-23}$, our results suggest that warming-induced increases in the relative abundance of potential plant pathogens in soils from natural ecosystems will increase the risk of infection by 
these fungi in adjacent croplands ${ }^{24-26}$. These impacts are likely to have implications for sustaining a growing human population, which is predicted to reach 9.8 billion people in $2050^{27}$. Furthermore, it can create significant constraints for livelihood in least developed countries, where the majority of people rely to a large degree on livestock and natural products supported by natural ecosystems ${ }^{10}$.

To experimentally corroborate the observed global patterns, we used a nine-year field warming experiment located at the centre of the Iberian Peninsula ${ }^{16}$, where natural ecosystems are expected to be markedly affected by global warming if emissions are not significantly controlled ${ }^{17}$. Note that these data were not included in our global survey and were analysed independently. This 140 experiment evaluates the effects of warming $\left(\sim 2^{\circ} \mathrm{C}\right.$; Supplementary Fig. 6) on key ecosystem attributes in a semiarid grassland with well-developed biocrusts (soil surface communities dominated by lichens, mosses, fungi, and cyanobacteria) ${ }^{16}$. Warming almost tripled the relative abundance of potential plant pathogens in soil (Fig. 3), providing additional experimental evidence of the positive effect of temperature on the relative abundance of these organisms. Additionally, 145 warming increased the relative (measured via amplicon-sequencing) and total (measured via quantitative PCR) abundance of Alternaria, the most common pathogenic fungal genus found in our global survey (Fig. 1) by sevenfold and twofold, respectively (Fig. 3). Warming also increased the relative abundance of the globally dominant Fusarium genus (Fig. 1) by almost five times (Supplementary Fig. 7), and also affected other common pathogens such as Cladosporium spp., where relative abundance increased by 20 -fold (see Supplementary Fig. 7 for more examples).

Global atlases, similar to those that have been available for plants and animals for centuries, now exist for some bacterial ${ }^{28}$ and fungal (e.g., mycorrhizal fungi) ${ }^{15,29}$ taxa. However, although regional and local information on plant diseases is starting to be increasingly available (https://www.plantwise.org), global atlases for the current and future distribution of potential plant pathogens under contrasting global change scenarios are lacking. Based on the consistent results from the global survey and experiment, we generated a global atlas depicting the current distribution of potential plant pathogens globally (Figs. 4A and Supplementary Figs. 8-9; see Supplementary Appendix 2 for a cross-validation on this map using an independent database ${ }^{9}$ ). We also generated a similar map for the relative abundance of potential pathogens with single 160 tropic mode (plant pathogens only) (Supplementary Fig. 9); this map is highly correlated to that including all potential plant pathogens together (Fig. 4A; Pearson's $\mathrm{r}=0.83 ; P<0.0001$ ). These atlases show that the highest relative abundance of these pathogens can be found in warm areas such as dryland and tropical ecosystems (Fig. 4A; Supplementary Fig. 9; Supplementary Appendixes 1-2). Analyses conducted for dominant potential plant pathogens revealed that while

165 Venturia has a more homogeneous spread across the globe, with especial relevance across the Northern Hemisphere, fungi from the genera Fusarium, Phoma, and Alternaria are more prevalent in tropical forests and drylands (Supplementary Fig. 10). These results are consistent with findings from croplands, where disease severity associated with these fungi is often more significant in warmer climates ${ }^{7,30}$.

170 To provide new insights on other potential locations on Earth that might be more vulnerable to these organisms in the future, we forecasted the relative abundance of potential plant pathogens under global change scenarios (RCP2.6-SSP1, RCP6.0-SSP4, RCP8.5-SSP5 up to 2050; Fig. 4B and Supplementary Fig. 10). These analyses show an increase of the relative abundance of potential plant pathogens in most regions of the world regardless of the climate and land-use 175 scenarios considered (Fig. 4B). Such an increase is supported by our experimental results showing a positive correlation of the abundance of these pathogens with warming effects like those expected 
by global climate models. Although caution should be taken regarding the local accuracy of our model (see Supplementary Appendix 1), the impacts of warming are particularly evident in soils across the Northern Hemisphere, towards the Arctic, as well as in South Africa, where all scenarios show a systematic temperature rise (Fig. 4). Land use was especially important for some potential pathogenic genera such as Fusarium, which were found to be negatively correlated with plant cover (Fig. 2), and thus might increase with forecasted increases in aridity ${ }^{11}$. Together, our analyses show those locations of Earth where potential plant pathogens are expected to become more common in the near future. However, we would also like to stress here that we have not measured pathogen infection or disease of hosts, and that the importance of pathogens in determining vegetation structure might differ in warm vs. cold ecosystems, which might limit the implications of our results in boreal and artic ecosystems. In addition, our study has a global focus and does not provide high resolution information on the fine-scale (e.g. at the scale of meters or centimeters) distributions of fungal pathogens, which are affected by factors not included in our

190 analyses such as microclimatic variations. Therefore, future work needs to be done to identify the fine-scale distribution of plant pathogens in specific localities.

Our results, based on a global survey and a nine-year field experiment, highlight the significance of soils from natural ecosystems as an important reservoir for potential fungal plant pathogens, and underscore temperature as a major environmental factor driving their global distribution. They indicate that the proportion of potential plant pathogens will likely increase in most regions of the world regardless of the climate and land use scenarios considered. Our findings advance our understanding of the distribution and sensitivities to climate and land-use change of potential fungal plant pathogens in a warmer and human-dominated world. They can also be used to make better predictions on how ongoing global environmental change will affect their distribution and impact on food production and human livelihoods worldwide.

\section{References}

1. Barford E. Crop pests advancing with global warming. Nature doi:10.1038/nature.2013.13644 (2013).

205 2. Newbery F. et al. Modelling impacts of climate change on arable crop diseases: progress, challenges and applications. Current Opinion in Plant Biology 32, 101-109 (2016).

3. Tollefson J. IPCC says limiting global warming to $1.5^{\circ} \mathrm{C}$ will require drastic action. Nature 562, 172-173 (2018)

4. Chakraborty S. Newton A.C. Climate change, plant diseases and food security. Plant Pathology 60, 2-14 (2011).

6. Nguyen, N.H. et al. FUNGuild: An open annotation tool for parsing fungal community datasets by ecological guild. Fungal Ecology 20, 241-248 (2016).

7. Parry D.W. et al. Fusarium ear blight (scab) in small grain cereals - a review. Plant Pathology 44, 207-238 (1993).

215 8. Qiu Z. et al. New frontiers in agriculture productivity: Optimised microbial inoculants and in situ microbiome engineering. Biotechnology Advance (2019).

9. Tedersoo L. et al. Global diversity and geography of soil fungi. Science 346, 1256688 (2014).

10. Asner G.P. et al. Grazing systems, ecosystem responses, and global change. Annual Review of Environment and Resources 29, 261-299 (2004).

11. Maestre F.T. et al. Structure and functioning of dryland ecosystems in a changing world. Annual Review of Environment and Resources 47, 215-237 (2016). 
12. IPCC: Climate Change 2013: The Physical Science Basis (Cambridge University Press, Cambridge, NY, USA, 2013).

13. Oliverio A.M. et al. Identifying the microbial taxa that consistently respond to soil warming across time and space. Global Change Biology 23, 2117-2129 (2017).

14. Bebber D.P. et al. The global spread of crop pests and pathogens. Global Ecology and Biogeography 23, 1398-1407 (2013).

15. Egidi E. et al. A few Ascomycota taxa dominate soil fungal communities worldwide. Nature Communications 10, 2369 (2019).

16. De Guevara M.L. et al. The 'PhenoBox', a flexible, automated, open-source plant phenotyping solution. New Phytologist 10.1111/nph.15000 (2018).

17. Guiot J., Wolfgang Cramer W. Mediterranean warming fast, deserts may spread in Europe: scientific paper. Science 354, 465-468 (2016).

18. Dean R. et al. The Top 10 fungal pathogens in molecular plant pathology. Molecular Plant Pathology 13, 414-30 (2012).

19. Agrios, G.N. Plant Pathology (St. Louis, MO: Academic Press, Cambridge, USA, 2005).

20. IPCC Special Reports: Land Use, Land-Use Change and Forestry (Cambridge University Press, Cambridge, NY, USA, 2000).

21. Bell, T. Tylianakis J.M. Microbes in the Anthropocene: spillover of agriculturally selected bacteria and their impact on natural ecosystems. Proc Biol Sci. 283, 20160896 (2016).

22. Caliz $\mathbf{J}$ et al.A long-term survey unveils strong seasonal patterns in the airborne microbiome coupled to general and regional atmospheric circulations. Proc Natl Acad Sci U S A. 115, 12229-12234 (2018).

23. Barberan A. et al. Continental-scale distributions of dust-associated bacteria and fungi. Proc Natl Acad Sci U S A. 112, 5756-5761 (2015).

24. Sugden A.M. Warming, crops, and insect pests. Science 361, 888-889 (2018).

25. Borrelli P. et al.An assessment of the global impact of 21st century land use change on soil erosion. Nature Communications 8, 2013 (2017).

26. Panagos P. et al. The new assessment of soil loss by water erosion in Europe. Environmental Science and Policy 54, 438 (2015).

27. World Population Prospects 2019: Ten Key Findings (United Nations, Department of Economic and Social Affairs, Population Division, NY, USA, 2019).

28. Delgado-Baquerizo M. et al. A global atlas of the dominant bacteria found in soil. Science 325, 320-325 (2018).

255 29. Steidinger BS et al. Climatic controls of decomposition drive the global biogeography of forest-tree symbioses. Nature 569, 404-408 (2019).

30. Köhl J. et al. Epidemiology of dark leaf spot caused by Alternaria brassicicola and A. brassicae in organic seed production of cauliflower. Plant Pathology 59, 358-367 (2010).

Methods

Global survey

Study sites and soil sampling

We used data from a global field survey ${ }^{15}$ to identify the ecological drivers and the current and future distribution of potential soil-borne plant pathogens in soils worldwide. Briefly, bulk soils (top $7.5 \mathrm{~cm}$ ) were collected from 235 ecosystems located in 18 countries from six continents (Supplementary Fig. 1) and covering nine biomes (temperate, tropical and dry forests, cold, temperate, tropical and arid grasslands, shrubland, boreal) between 2003 and 2015. Locations were 
selected to provide a solid representation for most environmental conditions (e.g., climate, soil and vegetation types) found on Earth (Supplementary Appendix 1). For example, mean annual 270 precipitation and temperature in these locations ranged from 67 to $3085 \mathrm{~mm}$ and from $-11.4^{\circ}$ to $26.5^{\circ} \mathrm{C}$, respectively. Given the global distribution of croplands, most natural ecosystems are surrounded to certain level by agricultural fields. Soil samples were sieved upon arrival to the laboratory ( $2 \mathrm{~mm}$ mesh). Then, a portion of soil was immediately frozen at $-20{ }^{\circ} \mathrm{C}$ for molecular analyses, while the rest of the soil was air-dried, and stored for a month, before physicochemical 275 analyses.

\section{Environmental factors}

Our field global survey ${ }^{15}$ included 12 environmental variables, which were obtained either in the field or from satellites/databases. Elevation and climatic variables, including mean annual 280 temperature (MAT), mean annual precipitation (MAP) and temperature and precipitation seasonality, were collected from the Worldclim database (https://www.worldclim.org; $(\sim 1 \mathrm{~km}$ resolution) ${ }^{31}$. Note that air and soil (https://neo.sci.gsfc.nasa.gov/) temperature are highly correlated at the global scale (Pearson $\mathrm{r}=0.81, \mathrm{P}=0.0011$ ) and that we used air temperature because current and future global models for this variable are more robust. Plant cover (20012015) was obtained using remote sensing data from the Moderate Resolution Imaging Spectroradiometer (MODIS) at $\sim 1 \mathrm{~km}$ resolution ${ }^{32}$. Soil properties (texture [\% of clay $\left.+\mathrm{silt}\right], \mathrm{pH}$ and total organic C) were determined from topsoil (top $7.5 \mathrm{~cm}$ ) samples collected from each location using standardized protocols ${ }^{33}$. To avoid biases associated with having multiple laboratories analyzing soils from different sites, all samples were analyzed at the Universidad Rey 290 Juan Carlos (Spain). Soil pH was measured with a pH meter, in a 1: 2.5 mass: volume soil and water suspension. Soil texture (\% of fine fractions: clay + silt) was determined as detailed in ref. ${ }^{33}$. The concentration of soil total organic carbon (C) was determined using a wet chemistry method ${ }^{34}$. Statistical analyses

Structural Equation Modelling

295 We used Structural Equation Modelling (SEM) ${ }^{35}$ to identify the direct and indirect effects of climate, vegetation and soil properties as drivers of the relative abundance potential plant pathogens (see our a priori model in Supplementary Fig. 3). The most common vegetation types in our database (forests and grasslands) were included in our SEM as categorical variables with two levels: 1 (a given ecosystem type) and 0 (remaining ecosystem types). Since some of the $300 \quad$ variables introduced were not normally distributed, the probability that a path coefficient differs from zero was tested using bootstrap tests ${ }^{36}$. Bootstrapping is preferred to the classical maximumlikelihood estimation in these cases, because in bootstrapping, probability assessments are not based on an assumption that the data match a particular theoretical distribution. Thus, data are randomly sampled with replacement in order to arrive at estimates of standard errors that are 305 empirically associated with the distribution of the data in the sample. We conducted models for the relative abundance (\%) of all soil-borne fungal plant pathogens (un-rarefied and rarefied, 4500 reads/sample; see the Molecular analyses section below), plant pathogens with single (plant pathogens only) and mixed trophic mode (plant pathogen and endophyte and/or saprotrophic fungi) and plant pathogens classified as probable and highly probable plant pathogens (excluding possible pathogens) ${ }^{6}$. Moreover, we conducted models for the most abundant pathogen genera (Alternaria, Fusarium, Venturia, and Phoma). Environmental data included in our model (Supplementary Table 1) did not suffer from multicollinearity (Pearson's $r<0.7$ in all cases; Supplementary Table 10). 
We then tested the goodness of fit of our model. To do so, we used the Chi-square test $\left(\chi^{2}\right.$; the model has a good fit when $0 \leq \chi^{2} \leq 2$ and $\left.0.05<\mathrm{p} \leq 1.00\right)$ and the root mean square error of approximation (RMSEA; the model has a good fit when RMSEA $0 \leq$ RMSEA $\leq 0.05$ and $0.10<$ $\mathrm{p} \leq 1.00^{36}$. Finally, we confirmed the fit of the model using the Bollen-Stine bootstrap test (the model has a good fit when $0.10<$ bootstrap $\mathrm{p} \leq 1.00$ ). Our model showed a solid goodness-of-fit, and therefore, a satisfactory fit to our data (Fig. 1D). SEM models were conducted with the software AMOS 20 (IBM SPSS Inc, Chicago, IL, USA).

\section{Correlation analyses}

We conducted Spearman correlation analyses to further evaluate the associations between climate, vegetation, soil properties and the relative abundance of the most ubiquitous putative fungal plant pathogens (i.e. those genera found in $>50 \%$ of all locations surveyed). Spearman rank correlations measure the strength and direction of association between two ranked variables. They do not require normality of data, and linearity is not a strict assumption of these analyses. We used a False Discovery Rate approach to determine adjusted p-values for all correlations to control for spurious (false positives) correlations. We used the R package "fdrtool"37 to conduct these analyses.

\section{Global mapping and predictions}

We used the sampled dataset to generate global maps of likely distributions of these pathogens. In particular, we conducted ordinary least square models to project each map for current and future states of soil pathogens across the world. The implementation of these models was preceded by 335 exploratory correlation analyses to identify the most important factors associated with the distributions of potential plant pathogens. These included: climate (DMAT: mean annual temperature; DMAP: mean annual precipitation), vegetation type (Dforest: forest; Dgrassland: grassland), elevation (Selev) and soil variables (Stext: soil texture; Scarbon: soil carbon; SpH: soil $\mathrm{pH}$ ). ' $\mathrm{S}$ ' and ' $\mathrm{D}$ ' indicate the variables that were either kept constant for current and future 340 conditions (S) or those that changed in future scenarios (D). Climatic seasonality data was not included in these analyses given the current levels of projection uncertainty associated with this type of data under contrasting global change scenarios ${ }^{38}$.

For future projections of the relative abundance of potential plant pathogens, we used precipitation, temperature and land-use datasets from the Inter-Sectoral Impact Model 345 Intercomparison Project (ISIMIP) ${ }^{39}$, and the land-use Model Intercomparison Project (LUMIP) ${ }^{40}$ activities from the Intergovernmental Panel for Climate Change (IPCC). This selection followed the protocol laid out in ref $^{41}$.

In terms of climate datasets, we used the bias-corrected historical and future ISMIP2a dataset $^{39,42}$ spanning the timeframe from 1951 to 2099 . We considered three Representative

$350 \quad$ Concentration Pathways: RCP2.6 ( +0.4 to $1.6^{\circ} \mathrm{C}$ by 2050$)$, RCP6.0 ( +0.8 to $1.8^{\circ} \mathrm{C}$ by 2050$)$, and RCP8.5 ( +1.4 to $2.6^{\circ} \mathrm{C}$ by 2050$)$. The monthly means of daily temperatures and daily total precipitation greater than $1 \mathrm{~mm}$ were calculated for the available period of these data. For the purpose of this study, we selected two projection steps: 2010 and 2050. To avoid outliers, we calculated 20-year climatologies using an analysis window centered in each year-step. The dataset created was used as a climate input for all model runs. For each SSPXRCP combination, we used two different general circulation models (GCM) (i.e., gfdl-esm2m, noresm1-m) ${ }^{42}$.

For the land-use projections, we built on the dataset provided by the land-use Harmonized v2.0 project (http://luh.umd.edu/) ${ }^{43}$. This dataset was produced in the context of the World Climate Research Program Coupled Model Intercomparison Project 6 (CMIP6) ${ }^{44-45}$, and contains a 
harmonized set of land-use scenarios that are consistent between historical reconstructions and future projections. It reproduces annual land-use reconstructions for historical land-use forcing (covering the period 850-2015) and for different integrated assessment models (IAMs) and shared socioeconomic pathways (SSP, from 2015 to 2100 ) at 0.25 degree resolution. These pathways represent a range of plausible future scenarios based on different socioeconomic challenges for climate change mitigation (low in SSP1 [sustainability] and SSP 4 [Regional inequality]; high in SSP5 [Fossil-fuelled development]), and potential challenges for adaptation (low in SSP1 and SSP5; high in SSP4). A full description of each scenario is given in ref. ${ }^{44}$. Each SSP corresponds to a specific RCP; here we selected the combinations SSP1xRCP2.6, SSP4xRCP6.0, and SSP5xRCP8.5. For the static datasets, we resampled all soil data coming from soil grids ${ }^{39}$ to 0.25 degree resolution to match the resolution of the non-static datasets. The same procedure was done with the elevation dataset ${ }^{46}$.

Using an exploratory analysis, which loops through all potential variable combinations to maximize the predicted power of each equation, we obtained different equations for each of the analysis:

$$
\begin{aligned}
P_{\text {Pathogens }}= & 0.905+\left(0.014 \times D_{M A T}\right)+\left(-<0.0011 \times D_{M A P}\right)+\left(0.194 \times D_{\text {forest }}\right) \\
& +\left(0.119 \times D_{\text {grassland }}\right)+\left(0.035 \times S_{\text {text }}\right)+\left(-0.295 \times S_{\text {carbon }}\right)+( \\
& \left.<0.0011 \times S_{\text {elev }}\right) \\
P_{\text {Alternaria }}=- & 0.194+\left(0.012 \times D_{M A T}\right)+\left(-0.052 \times D_{\text {forest }}\right)+\left(0.010 \times D_{\text {grassland }}\right) \\
& +\left(-0.113 \times S_{\text {text }}\right)+\left(0.913 \times S_{p H}\right)+\left(-0.313 \times S_{\text {carbon }}\right) \\
& +\left(<0.0011 \times S_{\text {elev }}\right) \\
P_{\text {Fusarium }}=- & 0.013+\left(<0.0012 \times D_{M A P}\right)+\left(0.117 \times D_{\text {grassland }}\right)+\left(0.409 \times S_{p H}\right) \\
& +\left(-0.310 \times S_{\text {carbon }}\right)+\left(<0.0011 \times S_{\text {elev }}\right) \\
P_{\text {Phoma }}=-0.483+\left(0.009 \times D_{M A T}\right)+\left(-<0.0011 \times D_{M A P}\right)+\left(0.175 \times D_{\text {forest }}\right) & +\left(0.014 \times D_{\text {grassland }}\right)+\left(0.699 \times S_{p H}\right)+\left(-0.029 \times S_{\text {text }}\right)+(- \\
& \left.<0.00104 \times S_{\text {elev }}\right) \\
P_{\text {Venturia }}=0.400+\left(0.008 \times D_{M A T}\right)+\left(-<0.0012 \times D_{M A P}\right)+\left(0.162 \times D_{\text {forest }}\right) & +\left(0.041 \times S_{\text {text }}\right)+\left(-0.585 \times S_{p H}\right)+\left(0.173 \times S_{\text {carbon }}\right)
\end{aligned}
$$

The equations mentioned above translate to different fit parameters: i) all potential plant pathogens (PPathogens): $\mathrm{R}^{2}=0.16, \mathrm{P}<0.001$; ii) Alternaria $\left(\mathrm{P}\right.$ Alternaria): $\mathrm{R}^{2}=0.27, \mathrm{P}<0.001$; iii) Fusarium (PFusarium): $\mathrm{R}^{2}=0.18, \mathrm{P}<0.001$; iv) Phoma (PPhoma): $\mathrm{R}^{2}=0.37, \mathrm{P}<0.001$; and vi) Venturia (PVenturia): $\mathrm{R}^{2}=0.26, \mathrm{P}<0.05$. A map of the extrapolation uncertainty for our global database (235 locations) is available in Supplementary Fig. 8 (see also Supplementary Appendix

395 1). In addition, we further cross-validated our main map using an independent global database as explained in Supplementary Appendix 2 below.

\section{Field experiment}

\section{Study site and soil sampling}

$400 \quad$ We used a nine-year manipulative field experiment to provide further experimental evidence for a causal link between warming and the relative abundance of soil-borne fungal potential plant pathogens. This experiment is being conducted on a dryland ecosystem located in the center of the Iberian Peninsula (4001'55.7"N 332'48.3"W; 590 m.a.s.1.). Mean annual temperature and rainfall are $15^{\circ} \mathrm{C}$ and $349 \mathrm{~mm}$, respectively and the soil is classified as Gypsiric Leptosol (IUSS Working 
Group WRB, 2006). Perennial plant cover is lower than $40 \%$, and is dominated by the perennial grass Stipa tenacissima L. Open areas between plant patches contain a well-developed biocrust community dominated by lichens such as Diploschistes diacapsis, Squamarina lentigera and Psora decipiens. Biocrust communities have been proposed as a system-model to test the effects of global change on ecosystem functioning under global change scenarios ${ }^{47-50}$. The experiment, described in ref. ${ }^{16}$, was established in the study area in July $2008^{50}$, and includes two levels of warming (ambient [control] vs. $\sim 2^{\circ} \mathrm{C}$ increase [warming] ${ }^{16,50}$.

To achieve a temperature increase within the forecasts of climate change models for the study area $^{51}$, we built open top chambers (OTCs) of hexagonal design with sloping sides of $40 \mathrm{~cm}$ $\times 50 \mathrm{~cm} \times 32 \mathrm{~cm}$ in $1.2 \times 1.2 \mathrm{~m}$ plots (Supplementary Fig. 6). We used methacrylate to build our OTCs because this material does not substantially alter the characteristics of the light spectrum. Our warming treatment promoted an average increase of air and surface soil $(0-2 \mathrm{~cm})$ temperature of $1.94^{\circ} \mathrm{C}$ and $2.55^{\circ} \mathrm{C}$, respectively. Warming effects were highest during the summer (JuneSeptember).

Soil samples (top 0-1 cm depth) were collected nine years after the beginning of the experiment from ten plots per combination of treatments. Three soil samples per plot were sampled with a $5 \mathrm{~cm}$ diameter core, which were then bulked to obtain a unique sample per plot. Soil was sieved ( $2 \mathrm{~mm}$ mesh) and separated into two fractions. A portion of soil was immediately frozen at $-20^{\circ} \mathrm{C}$ for molecular analyses. Given the different soil sampling depth between our experimental and observational study, caution should potentially be applied when directly comparing the two datasets.

We used non-parametric PERMANOVA (Anderson 2001) to test for significant effects of warming on the (ITS amplicon sequencing and qPCR analyses) abundance of fungal plant pathogens (see the Molecular analyses section below). These analyses are robust to lack of normality in our data. Warming was considered a fixed factor in these analyses $(\mathrm{n}=10)$. Non430 metric PERMANOVA analyses were carried out using PRIMER v 6113 and PERMANOVA ${ }^{+}$ (PRIMER-E, Plymouth, UK).

\section{Molecular analyses}

Amplicon sequencing

435 Amplicon sequencing analyses were used to determine the fungal communities in soils from the global survey and warming experiment. The extracted DNA samples were frozen and shipped to the Next Generation Genome Sequencing Facility of the University of Western Sydney (Australia). Fungal communities were determined by sequencing the Internal Transcribed Spacer (ITS) region 2 with primers FITS7 (GTGARTCATCGAATCTTTG) /ITS4 (TCCTCCGCTTATTGATATGC) 440 on a Illumina MiSeq platform $(2 \times 300 \mathrm{PE})$. Bioinformatic processing was performed using a combination of USEARCH ${ }^{52}$ and UNOISE3 ${ }^{53}$. Operational taxonomic units or OTUs (phylotypes) were defined at $100 \%$ similarity thresholds using UNOISE $3^{53}$. Phylotype identification was obtained against the UNITE fungal database $(\text { V7.2 })^{54}$. The relative abundance (\%) of each phylotype was calculated from the resulting OTU (phylotype) table. Plant pathogenic lifestyles for 445 fungal communities were determined using the FUNGuild database (http://www.stbates.org/guilds/app.php; retrieved at September 2019) ${ }^{6}$. A complete list of the potential soil-borne fungal plant pathogens included in this study can be found at Supplementary Data 1 (supplementary Excel file). We obtained 12086669 (global survey; $\mathrm{n}=235$ ) and 787142 (field experiment; $\mathrm{n}=20$ ) ITS reads across the studied samples, being $14.4 \%$ and $21.6 \%$ of all the retrieved ITS reads classified as putative fungal plant pathogens in the global survey and field 
experiment, respectively. The relative abundance of all soil-borne fungal plant pathogens (both exclusively pathogenic or with mixed life styles) was calculated in both cases using un-rarefied ITS OTU tables, as the sum of the relative abundance $(\%)$ of all ITS sequences classified as fungal plant pathogens (i.e., sum of all ITS reads classified as pathogens / all ITS reads x 100 at each soil sample). The total relative abundance of potential plant pathogens was highly correlated with the same variable calculated using a rarefied OTU table for the global field survey (4500 reads/sample; $\mathrm{r}=0.998 ; P<<0.0011)$ and the field experiment (4500 reads/sample; $\mathrm{r}=0.999 ; P<<0.0011$ ), so the choice of not rarefying our data did not affect our conclusions. All Gibberella reads were considered as Fusarium in this study for consistency with the most recent classifications ${ }^{55}$.

Additional taxonomic assignment analyses

To further confirm the robustness of the taxonomic assignments, for each phylotype identified as a putative plant pathogen, we performed a BLAST search (https://blast.ncbi.nlm.nih.gov/Blast.cgi) against the fungal ITS sequences from type material, and representative fungal genomes available in GenBank (https://www.ncbi.nlm.nih.gov/genbank/). We then selected the top 10 hits for each phylotype, and then re-parsed those matching species with FUNGuild. A total of 1574 and 586 OTUs matched, at a 97\% identity cut-off, with ITS ex-type sequences and representative genomes, respectively (Supplementary Data 1), having pathogenic trophic modes (both exclusively pathogenic and mixed modes). The relative abundance of all plant pathogens identified using the UNITE fungal database (V7.2) ${ }^{54}$ was highly correlated to the one calculated using GenBank from ITS ex-type ( $\mathrm{r}=0.96 ; P<<0.0011 ; 97 \%$ cut-off) and representative genomes $(\mathrm{r}=0.71 ; P<$ $<0.0011 ; 97 \%$ cut-off) (Supplementary Data 1). These analyses provide further support of our data.

\section{qPCR analyses}

475 qPCR analyses were done to further confirm results from our warming experiment. The absolute abundance of Alternaria -the most predominant fungal plant pathogen in our surveys- was estimated by a real-time quantitative polymerase chain reaction (qPCR) using primers Dir1ITSAlt (TGTCTTTTGCGTACTTCTTGTTTCCT) and Inv1ITSAlt (CGACTTGTGCTGCGCTC), which are commonly used to quantify pathogenic plant-associated Alternaria $\operatorname{spp}^{56}$. Mastermix reactions 480 were prepared in a volume of $10 \mu \mathrm{l}$ containing a $1.5 \mathrm{ng}$ DNA template, $5 \mu \mathrm{l} 2 \times$ SensiFast SYBR Hi-ROX kit (Bioline, Australia), $2 \mu \mathrm{l}$ water and $1 \mu \mathrm{l}(5 \mu \mathrm{mol} / \mu \mathrm{l})$ of each primer, respectively. Amplifications were performed in 96-well reaction plates using a Bio-RAD CFX96 real-time PCR system (Bio-Rad, Australia). Each plate included duplicate reactions per DNA sample, standards and a negative control sample (without DNA). Standard curves were generated using tenfold serial 485 dilution of PCR-amplicons containing the Alternaria target region. The amplification program consisted of 1 cycle of $94^{\circ} \mathrm{C}$ for $4 \mathrm{~min}$, followed by 35 cycles of $94^{\circ} \mathrm{C}$ for $30 \mathrm{~s}, 62^{\circ} \mathrm{C}$ for $25 \mathrm{~s}$ and $72^{\circ} \mathrm{C}$ for $20 \mathrm{~s}$, and a final elongation step of $72{ }^{\circ} \mathrm{C}$ for $2 \mathrm{~min}$. To determine the reaction specificity a melting curve analysis was subsequently performed by incubating the samples at $95^{\circ} \mathrm{C}$ for $2 \mathrm{~min}$, annealing at $65^{\circ} \mathrm{C}$ for $5 \mathrm{~s}$, followed by heating them slowly at $0.5^{\circ} \mathrm{C} / \mathrm{sec}$ up to $95^{\circ} \mathrm{C}$, while $490 \quad$ continuously monitoring the fluorescence signal.

\section{Methods references}

31. Hijmans, R.J. et al. Very high resolution interpolated climate surfaces for global land areas Int. J. of Clim. 25, 1965-1978 (2005). 
32. Filipponi F. et al. Global MODIS fraction of green vegetation cover for monitoring abrupt and gradual vegetation changes. Remote Sens. 10, 653 (2018).

33. Maestre F.T. et al. Plant species richness and ecosystem multifunctionality in global drylands. Science 335, 214-218 (2012).

34. Anderson J.M., Ingramm J.S.I., Eds., Tropical Soil Biology and Fertility: A Handbook of Methods (CABI, Wallingford, UK, ed. 2, 1993).

35. Grace J.B. Structural Equation Modeling Natural Systems (Cambridge Univ. Press, Cambridge, 2006).

36. Schermelleh-Engel, K. et al. Evaluating the Fit of Structural Equation Models: Tests of Significance and Descriptive Goodness-of-Fit Measures. Methods Psychol. Res. 8, 23-74 (2003).

37. Klaus B., Strimmer K. Estimation of (Local) False Discovery Rates and Higher Criticism. R package "fdrtool" (https://cran.r-project.org/), Version 1.2.15 (2015).

38. Monteleoni, C. et al. Tracking climate models Stat Anal Data Min. 4, 372-392 (2011).

39. Hempel S. et al. A trend-preserving bias correction - the isi-mip approach. Earth System Dynamics. 4, 219-236 (2013).

40. Lawrence D. M. et al. The Land Use Model Intercomparison Project (LUMIP) contribution to CMIP6: rationale and experimental design. Geoscientific Model Development 9, 2973-2998 (2016).

41. Kim H. et al. A protocol for an intercomparison of biodiversity and ecosystem services models using harmonized land-use and climate scenarios. Geoscientific Model Development 11, 45374562 (2018).

42. Dufresne J.-L. et al. Climate change projections using the IPSL-CM5 Earth System Model: from CMIP3 to CMIP5. Clim. Dyn. 40, 2123-2165 (2013).

43. Hurtt G.C. et al. Harmonization of land-use scenarios for the period 1500-2100: 600 years of global gridded annual land-use transitions, wood harvest, and resulting secondary lands. Clim. Change. 109, 117 (2011).

44. Popp A. et al. Land-use futures in the shared socio-economic pathways. Glob. Environ. Change. 42, 331-345 (2017).

525 45. O'Neill B.C. et al.A new scenario framework for climate change research: the concept of shared socioeconomic pathways. Climatic Change. 122, 387-400 (2014).

46. Global Multi-resolution Terrain Elevation Data 2010 (GMTED2010) | The Long Term Archive, (available at https://ta.cr.usgs.gov/GMTED2010).

$530 \quad$ Crusts: An Organizing Principle in Drylands (Springer, Berlin, Germany, 2016).

48. Bowker M.A. et al. Biological soil crusts (biocrusts) as a model system in community, landscape and ecosystem ecology. Biodivers Conserv 23,1619-1637 (2014).

49. Castillo-Monroy A.P. et al. Biological soil crusts modulate nitrogen availability in semi-arid ecosystems: Insights from a Mediterranean grassland. Plant and Soil 333, 21-34 (2010).

535 50. Maestre F.T. et al. Changes in biocrust cover drive carbon cycle responses to climate change in drylands. Global Change Biology 19, 3835-3847 (2013).

51. De Castro $M$ et al. Evaluación Preliminar de los Impactos en España por Efecto del Cambio Climático (Ministerio Medio Ambiente, Madrid, Spain, 2005).

540 2460-2461 (2010). 
53. Edgar, R.C. UNOISE2: improved error-correction for Illumina 16S and ITS amplicon sequencing. BioRxiv 081257. DOI: 10.1101/081257 (2016).

54. Kõljalg, U., et al. UNITE: a database providing web-based methods for the molecular identification of ectomycorrhizal fungi. New Phytologist 166, 1063-1068 (2005).

55. Geiser D.M. et al. One fungus, one name: Defining the genus Fusarium in a scientifically robust way that preserves longstanding use. Phytopathology 103, 400-408 (2013).

56. Kulik, T. et al. Quantification of Alternaria, Cladosporium, Fusarium and Penicillium verrucosum in Conventional and Organic Grains by qPCR. Journal of Phytopathology 163, 522-528 (2015).

57. Delgado-Baquerizo, M. et al. The proportion of soil-borne pathogens increases with warming at the global scale. Figshare digital repository. DOI: 10.6084/m9.figshare.11484747 (2019).

\section{Correspondence statement}

*Correspondence to: Manuel Delgado-Baquerizo. E-mail: M.DelgadoBaquerizo@gmail.com

\section{Acknowledgements}

This project received funding from the European Union's Horizon 2020 research and innovation programme under the Marie Sklodowska-Curie grant agreement No 702057 and the European Research Council (ERC) grant agreements no. 242658 (BIOCOM) and 647038 (BIODESERT). We would like to thank Richard D. Bardgett, Noah Fierer, Alberto Benavent-González and David J. Eldridge for their original contributions to the global survey, and Victoria Ochoa, Cristina Escolar, Patricia Alonso, Beatriz Gozalo and Sergio Ochoa for maintaining the warming experiment and for their help with laboratory analyses. We also thank Melissa Martin for revising the English of the manuscript. M.D-B. is supported by the MUSGONET grant (LRA17\1193) from the British Ecological Society. FTM also acknowledges funding from Generalitat Valenciana (CIDEGENT/2018/041) and from sDiv, the synthesis center of German Centre for Integrative Biodiversity Research Halle-Jena-Leipzig (iDiv). Work on microbial distribution and colonisation in BKS laboratory is funded by Australian Research Council (DP190103714). BKS also acknowledges a research award by the Humboldt Foundation. NE acknowledges support from iDiv, funded by the German Research Foundation (FZT 118) Flexpool proposal 34600850, and from ERC under the European Union's Horizon 2020 research and innovation program (grant agreement no. 677232).

\section{Author contributions}

575 M.D-B. developed the original idea of the analyses presented in the manuscript. M.D-B, F.T.M., and B.K.S. led the global survey. F.T.M. designed the field warming experiment and has maintained it over the years. Lab analyses were done by M.D-B., C.C-D., E.E., F.T.M. and B.K.S. Bioinformatic analyses were done by B.K.S., J.W and E.E. Statistical modelling, mapping and data interpretations were done by C.G., N.E. and M.D-B. The manuscript was written by M.D-B. 580 with contributions from all co-authors.

\section{Data accessibility}

The data associated with the global field survey and the field experiment are publicly available in Figshare $^{57}$.

\section{Code availability}


Most numerical analyses included in this article do not have an associated code. Used codes are available in ref. ${ }^{57}$.

$590 \quad$ Competing financial interests

The authors declare no conflict of interest.

\section{Supplementary Information}

Supplementary Appendixes 1 to 2

Supplementary Figures 1 to 11

595 Supplementary Tables 1 to 10

600

605

610

615

620

625 


\section{Figure captions}

Figure 1 | Relative abundance, identity and ecological preferences of potential plant pathogens worldwide. Panel A represents the distribution of the relative abundance of total fungal pathogens across the 235 ecosystems surveyed. Panel B includes mean values $( \pm$ SE) for the relative abundance $(\%)$ of potential plant pathogens across continents/biomes. Panel $\mathrm{C}$ shows the most common soil fungal pathogens identified (mean values \pm SE). Panel D includes a structural equation model assessing the direct and indirect effects of environmental factors on the relative abundance of potential plant pathogens. We grouped the different categories of predictors (climate, soil properties, vegetation and spatial influence) in the same box for graphical simplicity (these boxes do not represent latent variables). Variable within these boxes are allowed to covary. Numbers adjacent to arrows are indicative of the effect size of the relationship. Only significant effects $(\mathrm{P}<0.05)$ are plotted. Information on environmental factors included in our SEM, and on direct effects for other SEM arrows can be found in Supplementary Fig. 3 and Supplementary Tables 1-2. Supplementary Table 2 offers a complete view of our full SEM. The degree of freedom in this SEM came from the lack of relationship between PSEA and clay+silt (\%). $\mathrm{R}^{2}$ for other endogenous variables in Supplementary Table 8. Panel E represents the total standardised effects on SEM (sum of direct and indirect effects; STE; \pm bootstrap CI 95\%) on the relative abundance of potential plant pathogens. In panels $A$ and $C-E, n=235$ locations. $n$ associated with panel $B$ are shown in parentheses. $\mathrm{F}=$ Forests; $\mathrm{G}=$ Grasslands. MAT = mean annual temperature. $\mathrm{MAP}=$ mean annual precipitation. PSEA = precipitation seasonality. TSEA = temperature seasonality.

$655 \quad$ Figure 2 | Temperature is positively associated with the relative abundance of potential plant pathogens at the genus level. Spearman correlations between environmental factors and the relative abundance of ubiquitous fungal plant pathogens at the genus level $(n=235)$. Information on environmental factors included in this analysis can be found in Supplementary Table 1. MAT $=$ mean annual temperature. $\mathrm{MAP}=$ mean annual precipitation. PSEA = precipitation seasonality. TSEA $=$ temperature seasonality. Correlations with False Discovery Rate adjusted $P>0.05$ are excluded (plotted in white).

Figure 3 | Experimental evidence that warming increases the relative and total abundance of potential plant pathogens. Warming effects on the relative (\%) and absolute (gene copies $\mathrm{g}^{-1}$ soil) abundance of fungal pathogens in a nine-year field warming experiment. The solid lines show mean values $(\mathrm{n}=10)$. $\mathrm{P}$ values as follows: $* * * \mathrm{P}<0.001 ; * \mathrm{P}<0.05$. ${ }^{*} \log _{10}$-transformed. See Supplementary Table 9 for further statistical details.

Figure 4 | Current relative abundance (A) and temporal projections (2050; B-C) of potential plant pathogens across the globe. A cross-validation of the map shown in A using an independent global survey is available in Supplementary Appendix 2. Panel B shows the agreement across the different scenarios considered (gain reflects areas where gain is predicted, loss reflects areas where loss is predicted, and mixed reflects areas where different scenarios predict gain or loss). Panel C shows the relative change for potential plant pathogens and that of the most abundant genera (Alternaria, Fusarium, Venturia, and Phoma) assessed for scenarios SSP1 (sustainability), SSP4 (Regional inequality), and SSP5 (Fossil-fueled development). The bars and barplots indicate the interquartile interval and median value for each scenario, respectively. A map of the extrapolation uncertainty for our global database (235 locations) is available in Supplementary Fig. 8 (see also 
Supplementary Appendix 1). See also Supplementary Figs. 9-10 for an alternative panel (A), and 680 for maps of individual pathogen-associated genera. 